PROCEEDINGS OF THE

AMERICAN MATHEMATICAL SOCIETY

Volume 126, Number 5, May 1998, Pages 1491-1500

S $0002-9939(98) 04525-0$

\title{
GLOBAL ASYMPTOTIC BEHAVIOR OF SOLUTIONS OF A SEMILINEAR PARABOLIC EQUATION
}

\author{
QI S. ZHANG AND Z. ZHAO
}

(Communicated by Jeffrey B. Rauch)

\begin{abstract}
We study the large time behavior of solutions for the semilinear parabolic equation $\Delta u+V u^{p}-u_{t}=0$. Under a general and natural condition on $V=V(x)$ and the initial value $u_{0}$, we show that global positive solutions of the parabolic equation converge pointwise to positive solutions of the corresponding elliptic equation. As a corollary of this, we recapture the global existence results on semilinear elliptic equations obtained by Kenig and $\mathrm{Ni}$ and by F.H. Lin and Z. Zhao. Our method depends on newly found global bounds for fundamental solutions of certain linear parabolic equations.
\end{abstract}

\section{INTRODUCTION}

We shall study the large time behavior of solutions for the following semilinear parabolic problem:

$$
\left\{\begin{array}{l}
\Delta u(x, t)+V(x) u^{p}(x, t)-u_{t}(x, t)=0, \quad(x, t) \in \mathbf{R}^{n} \times(0, \infty), \\
u(x, 0)=u_{0}(x), \quad x \in \mathbf{R}^{n}, n \geq 3 .
\end{array}\right.
$$

Here $\Delta$ is the Laplacian and $V=V(x)$ is a Green-tight function in $\mathbf{R}^{n}$, a notion introduced by one of us in [Zhao1]. As pointed out in that paper, this class of functions is a natural one to consider for semilinear elliptic problems. For the reader's convenience we recall the definition. We will present more properties and examples in remarks 1.2 and 1.3 below while referring to [Zhao1] for further details.

Definition 1.1. A Borel measurable function $U$ is called a Green-tight function in $\mathbf{R}^{n}$ if

$$
\lim _{r \rightarrow 0}\left[\sup _{x} \int_{|x-y| \leq r} \frac{|U(y)|}{|x-y|^{n-2}} d y\right]=\lim _{M \rightarrow \infty}\left[\sup _{x} \int_{|y| \geq M} \frac{|U(y)|}{|x-y|^{n-2}} d y\right]=0 .
$$

As a motivation to our study, we give a short historic account. Both the parabolic problem (1.1) and its elliptic counterpart (1.2) have been widely studied.

$$
\Delta u(x)+V(x) u^{p}(x)=0, \quad x \in \mathbf{R}^{n} .
$$

$\mathrm{Ni}[\mathrm{N}]$ and Kenig and $\mathrm{Ni}[\mathrm{KN}]$ proved existence of global positive solutions of the elliptic problem (1.2) under the assumption that $|V(x)| \leq C /|x|^{2+\epsilon}$ for $\epsilon>0$ and when $|x|$ is large. Lin $[\mathrm{L}]$ proved the existence theorem for (1.2) assuming $|V(x)| \leq \phi(|x|) /|x|^{2}$ for large $|x|$, where $\phi$ satisfies $\int_{L}^{\infty}(\phi(r) / r) d r<\infty$ for large

Received by the editors November 6, 1996.

1991 Mathematics Subject Classification. Primary 35K57.

(C) 1998 American Mathematical Society 
$L>0$. Lin's result is therefore a generalization of that of $\mathrm{Ni}$ and Kenig and $\mathrm{Ni}$. In the paper [Zhao1], one of us showed that (1.2) has infinitely many global positive solutions when $V$ is in the class of Green-tight functions, which encompasses all the cases discussed by Kenig, Lin and Ni. Moreover, Zhao's method is flexible enough to deal with (1.2) when $V$ is singular. Recently another of us [Zhang2] established an essentially optimal condition on $V=V(x, t)$ so that the parabolic problem (1.1) (with time-dependent $V$ ) has global positive solutions.

In this paper we will investigate the asymptotic behavior of the global solutions of (1.1). Our main result, stated in Theorem A below, not only serves as a bridge between the elliptic and parabolic problems but also recaptures those of Kenig, Lin, $\mathrm{Ni}$ and Zhao mentioned earlier.

Theorem A. Suppose $V$ is Green-tight and $u_{0} \in C^{2}\left(\mathbf{R}^{n}\right)$. Then the following conclusions are true.

(a) There exists a positive number $\alpha$ such that (1.1) has a global positive solution whenever $0<u_{0} \leq \alpha$.

(b) If $\Delta u_{0}$ is Green-tight and $0<\beta \leq u_{0} \leq \alpha$, then (1.1) has a global positive solution which converges pointwise to a positive solution of $\Delta u+V u^{p}=0$.

Remark 1.1. The existence part of Theorem A (part (a)) was proved as a special case in [Zhang2]. For completeness we will write down the existence result in section 2. The main task is then to prove part (b). Our method is based on newly established global Gaussian upper bounds for fundamental solutions of certain linear parabolic equations. We emphasize that the parameters in the bounds, which will be given in section 3, are independent of time. This fact is essential to the proof of part (b), which will take place in section 4 .

Remark 1.2. We will frequently use the following fact proved in [Zhao1]. If $V$ is Green-tight in $\mathbf{R}^{n}$, then

$$
\sup _{x} \int_{\mathbf{R}^{n}} \frac{|V(y)|}{|x-y|^{n-2}} d y<\infty .
$$

It is also noteworthy that Green-tight functions are allowed to have local singularities as functions in the Kato class $K_{n}^{\text {loc }}$, which contains $L_{l o c}^{p}, p>n / 2$.

Remark 1.3. As proven in [Zhao1], any bounded functions $K(x)$ satisfying $|K(x)| \leq$ $C /\left(1+|x|^{(2+\epsilon)}\right)$ with $\epsilon>0$ are Green-tight functions in $\mathbf{R}^{n}$; so are the functions $K$ in Lin's paper [L].

\section{Preliminaries}

First we briefly describe the existence result in [Zhang2], which forms a basis of part (a) of Theorem A in this paper.

Given $h>0, c>0$ and $V=V(x, t)$, write

$$
\begin{aligned}
N_{c, h}(V)= & \sup _{x, t} \int_{t-h}^{t} \int_{B\left(x, h^{1 / 2}\right)}|V(y, s)| G_{c}(x, t ; y, s) d y d s \\
& +\sup _{y, s} \int_{s}^{s+h} \int_{B\left(y, h^{1 / 2}\right)}|V(x, t)| G_{c}(x, t ; y, s) d x d t
\end{aligned}
$$

here and always $G_{c}(x, t ; y, s) \equiv \frac{1}{(t-s)^{n / 2}} \exp \left(-c \frac{|x-y|^{2}}{t-s}\right)$. 
Definition 2.1 ([Zhang2]). We say that a function $V$ is in the class $P^{\infty}$ if

$$
\lim _{h \rightarrow 0} N_{c, h}(V)=0, \quad \text { and } \quad N_{c, \infty}(V) \equiv \lim _{h \rightarrow \infty} N_{c, h}(V)<\infty, \quad \text { for all } \quad c>0 .
$$

The class $P^{\infty}$ is quite rich. In particular $P^{\infty}$ contains the class of Green-tight functions, as we will prove in Proposition 2.1 below.

Theorem 2.1 ([Zhang2]). Suppose $p>1$ and $V=V(x, t) \in P^{\infty}$. For any $M>1$, there is a constant $b_{0}>0$, such that for each non-negative $u_{0} \in C^{2}\left(\mathbf{R}^{n}\right)$ satisfying $\left\|u_{0}\right\|_{L^{\infty}\left(\mathbf{R}^{n}\right)} \leq b_{0}$, there exists a positive and continuous solution $u$ of (1.1) such that

$$
M^{-1} \int_{\mathbf{R}^{n}} G_{0}(x, t ; y, 0) u_{0}(y) d y \leq u(x, t) \leq M \int_{\mathbf{R}^{n}} G_{0}(x, t ; y, 0) u_{0}(y) d y
$$

for all $(x, t) \in \mathbf{R}^{n} \times(0, \infty)$. Here $G_{0}$ is the fundamental solution of the heat equation $\Delta u-u_{t}=0$.

Proposition 2.1. The class $P^{\infty}$ contains the class of Green-tight functions in $\mathbf{R}^{n}$. Moreover, if $V=V(x)$, then $N_{c, \infty}(V) \leq C \sup _{x} \int_{\mathbf{R}^{n}} \frac{|V(y)|}{|x-y|^{n-2}} d y, c>0$.

Proof. This was done in [Zhang2], so we will be brief. Suppose $V=V(x)$ is a Green-tight function in $\mathbf{R}^{n}$; then $V \in K_{n}^{l o c}$ and $\sup _{x \in \mathbf{R}^{n}} \int_{\mathbf{R}^{n}} \frac{|V(y)|}{|x-y|^{n-2}} d y<\infty$ (see [Zhao1]). Therefore

$$
\begin{aligned}
\sup _{x, t} & \int_{-\infty}^{t} \int_{\mathbf{R}^{n}}|V(y)| G_{c}(x, t ; y, s) d y d s \\
& =\sup _{x, t} \int_{\mathbf{R}^{n}}|V(y)| \int_{-\infty}^{t} G_{c}(x, t ; y, s) d s d y \leq C \sup _{x} \int_{\mathbf{R}^{n}} \frac{|V(y)|}{|x-y|^{n-2}} d y<\infty .
\end{aligned}
$$

This shows that $N_{c, \infty}(V)<\infty$ for all $c>0$. q.e.d.

\section{Global Gaussian upper bounds}

In this section we establish global Gaussian upper bounds for the fundamental solutions of certain linear parabolic equations. This result was proved in [Zhang3] in a much more general context. Here we give a shorter and more direct proof.

Theorem 3.1. Let $G$ be the fundamental solution of the equation

$$
\Delta u(x, t)-u_{t}(x, t)+V(x, t) u(x, t)=0 .
$$

Suppose $N_{c, \infty}(V)$ is sufficiently small for a suitable $c>0$. Then there exist positive constants a and $C$ such that

$$
G(x, t ; y, 0) \leq \frac{C}{t^{n / 2}} \exp \left(-a|x-y|^{2} / t\right)
$$

for all $t>0$ and $x, y \in \mathbf{R}^{n}$.

Corollary 3.1. If $V=V(x)$ is Green-tight and $\int_{\mathbf{R}^{n}} \frac{|V(y)|}{|x-y|^{n-2}} d y$ is small, then the conclusion of Theorem 3.1 holds.

Proof of the Theorem. Without any loss of generality we assume that $V$ is bounded and supported in $\mathbf{R}^{n} \times[0, T]$, where $T$ is a positive number. This is because the general case can be covered by an easy limiting argument. What is important is to make sure all constants are independent of $T$. 
We pick a number $a$ such that $0<a<1 / 4$ and let $B$ be the smallest positive number such that

$$
G(x, t ; y, s) \leq B G_{a}(x, t ; y, s) \equiv B \frac{1}{t^{n / 2}} \exp \left(-a|x-y|^{2} / t\right)
$$

for all $x, y \in \mathbf{R}^{n}$ and $s<t$. We claim that such a $B$ does exist by our extra assumption that $V(x, t)=0$ if $t>T$. The claim can be checked easily by using the reproducing formula $G(x, t ; y, 0)=\int_{\mathbf{R}^{n}} G(x, t ; z, T) G(z, T ; y, 0) d z, \quad t>T$, and the fact that $G(x, t ; z, T)=G_{0}(x, t ; z, T)$ for $t>T$, where $G_{0}=C_{0} G_{1 / 4}$ is the fundamental solution of the heat operator $\Delta-\partial_{t}$. The main task is to show that $B$ depends on $V$ only in the form of $N_{c, \infty}(V)$.

By Duhamel's principle, we have

$$
\begin{aligned}
G(x, t ; y, s)= & G_{0}(x, t ; y, s)+\int_{s}^{t} \int_{\mathbf{R}^{n}} G(x, t ; z, \tau)|V(z, \tau)| G_{0}(z, \tau ; y, s) d z d \tau, \\
G(x, t ; y, s) \leq & G_{0}(x, t ; y, s) \\
& \quad+B C_{0} \int_{s}^{t} \int_{\mathbf{R}^{n}} G_{a}(x, t ; z, \tau)|V(z, \tau)| G_{1 / 4}(z, \tau ; y, s) d z d \tau,
\end{aligned}
$$

where $x, y \in \mathbf{R}^{n}$ and $s<t$. Lemma 5.1 in the appendix with $b=1 / 4$ then implies

$$
G(x, t ; y, s) \leq C_{0} G_{1 / 4}(x, t ; y, s)+B C_{0} C_{a, b} N_{c, \infty}(V) G_{a}(x, t ; y, s)
$$

for all $x, y \in \mathbf{R}^{n}$ and $s<t$. Since $a<1 / 4$, we know that

$$
G(x, t ; y, s) \leq\left[C_{0}+B C_{0} C_{a, b} N_{c, \infty}(V)\right] G_{a}(x, t ; y, s)
$$

for all $x, y \in \mathbf{R}^{n}$ and $s<t$. Hence, by the definition of $B$, we obtain

$$
B \leq C_{0}+B C_{0} C_{a, b} N_{c, \infty}(V) .
$$

When $C_{0} C_{a, b} N_{c, \infty}(V)<1 / 2$ we have

$$
B \leq 2 C_{0} \text {. q.e.d. }
$$

\section{Proof of the theorem}

Proof of Theorem A. Step 1. First we recall the conditions imposed on the initial function $u_{0}$ :

$$
u_{0} \in C^{2}\left(\mathbf{R}^{n}\right), \quad 0<\beta \leq u_{0} \leq \alpha, \quad \sup _{x} \int_{\mathbf{R}^{n}}\left|\Delta u_{0}(y)\right| /|x-y|^{n-2} d y<\infty,
$$

where $\alpha$ is a small number so that (1.1) has global positive solutions by Theorem 2.1. The reason to impose a positive lower bound $\beta$ for $u_{0}$ is to guarantee that the equilibrium solution is non-trivial. As pointed out in Remark 1.3, the condition on $\Delta u_{0}$ is satisfied if $\left|\Delta u_{0}(y)\right| \leq C /\left(1+|y|^{q}\right)$ with $q>2$. Since $V$ is also Green-tight by assumption, we have, by Remark 1.2 ,

$$
\sup _{x} \int_{\mathbf{R}^{n}}\left[\left|\Delta u_{0}(y)\right|+|V(y)|\right] /|x-y|^{n-2} d y<\infty .
$$

Step 2 . We temporally assume that $V$ is smooth. Let us write $w=u_{t}$. Differentiating the equation in (1.1), we have that $w$ solves

$$
\left\{\begin{array}{l}
\Delta w(x, t)-w_{t}(x, t)+p V(x) u^{p-1}(x, t) w(x, t)=0, \quad(x, t) \in \mathbf{R}^{n} \times(0, \infty), \\
w(x, 0)=\Delta u_{0}(x)+V(x) u_{0}^{p}(x) .
\end{array}\right.
$$


Let $V_{1} \equiv p V u^{p-1}$ and let $G_{1}$ be the fundamental solution of the operator $\Delta-\partial_{t}+V_{1}$. Then

$$
w(x, t)=\int_{\mathbf{R}^{n}} G_{1}(x, t ; y, 0)\left[\Delta u_{0}(y)+V(y) u_{0}^{p}(y)\right] d y .
$$

When $u_{0}$ is small, we know by Theorem 2.1 that $u$ is small, and so by Proposition 2.1

$$
N_{c, \infty}\left(V_{1}\right) \leq C p \sup |u|^{p-1} N_{c, \infty}(V) \leq C p \sup |u|^{p-1} \sup _{x} \int_{\mathbf{R}^{n}} \frac{|V(y)|}{|x-y|^{n-2}} d y
$$

is small. By Theorem 3.1 (global Gaussian upper bound), there exist positive constants $a, C$ independent of $t, x$ and $y$ such that

$$
G_{1}(x, t ; y, 0) \leq \frac{C}{t^{n / 2}} \exp \left(-a|x-y|^{2} / t\right)
$$

Therefore

$$
\left|u_{t}(x, t)\right| \leq \int_{\mathbf{R}^{n}} \frac{C}{t^{n / 2}} \exp \left(-a|x-y|^{2} / t\right)\left[\left|\Delta u_{0}(y)\right|+\left|V(y) u_{0}^{p}(y)\right|\right] d y .
$$

For any $t>0$, on integrating the above inequality from $t$ to $\infty$ we obtain, via Fubini's Theorem and (4.2),

$$
\int_{t}^{\infty}\left|u_{s}(x, s)\right| d s \leq C \int_{\mathbf{R}^{n}} \frac{1}{|x-y|^{n-2}}\left[\left|\Delta u_{0}(y)\right|+\left|V(y) u_{0}^{p}(y)\right|\right] d y<\infty .
$$

Here we have used the fact that

$$
\int_{t}^{\infty} \frac{1}{s^{n / 2}} \exp \left(-a|x-y|^{2} / s\right) d s \leq \frac{C}{|x-y|^{n-2}} .
$$

Now we have the pointwise convergence

$$
u_{\infty}(x) \equiv \lim _{t \rightarrow \infty} u(x, t)
$$

We claim that the rate of convergence in (4.7) depends only on $x$ and the rate of convergence of the following limit:

$$
\lim _{M \rightarrow \infty} \sup _{x} \int_{|y| \geq M} \frac{\left|\Delta u_{0}(y)\right|+|V(y)|}{|x-y|^{n-2}} d y=0
$$

which appears in Definition 1.1 of Green-tight functions. Here is a proof of the claim. Since $\Delta u_{0}$ and $V$ are Green-tight, we can find, for a fixed $x$ and any $\epsilon>0$, an $M>0$ such that

$$
\int_{|x-y| \geq M} \frac{\left|\Delta u_{0}(y)\right|+|V(y)|}{|x-y|^{n-2}} d y<\epsilon / 2 .
$$


From (4.5), we have

$$
\begin{aligned}
\mid u(x, t)- & u_{\infty}(x)\left|\leq \int_{t}^{\infty}\right| u_{s}(x, s) \mid d s \\
\leq & C \int_{t}^{\infty} \int_{\mathbf{R}^{n}} \frac{1}{s^{n / 2}} \exp \left(-a|x-y|^{2} / s\right)\left[\left|\Delta u_{0}(y)\right|+|V(y)|\right] d y \\
= & C \int_{t}^{\infty} \int_{|x-y| \leq M} \frac{1}{s^{n / 2}} \exp \left(-a|x-y|^{2} / s\right)\left[\left|\Delta u_{0}(y)\right|+|V(y)|\right] d y \\
& +C \int_{t}^{\infty} \int_{|x-y| \geq M} \frac{1}{s^{n / 2}} \exp \left(-a|x-y|^{2} / s\right)\left[\left|\Delta u_{0}(y)\right|+|V(y)|\right] d y \\
\leq & C \int_{t}^{\infty} \int_{|x-y| \leq M} \frac{1}{s^{n / 2}} \frac{M^{n-2}}{|x-y|^{n-2}}\left[\left|\Delta u_{0}(y)\right|+|V(y)|\right] d y \\
& +C \int_{|x-y| \geq M} \frac{1}{|x-y|^{n-2}}\left[\left|\Delta u_{0}(y)\right|+|V(y)|\right] d y \\
\leq & \frac{C}{t^{(n-2) / 2}} M^{n-2}+\epsilon / 2<\epsilon
\end{aligned}
$$

when $t$ is sufficiently large. This proves the claim.

If we use the obvious inequality $\frac{1}{t^{(n-2) / 2}} \exp \left(-a|x-y|^{2} / t\right) \leq C /|x-y|^{n-2}$ on (4.5), we obtain a pointwise estimate on $\left|u_{t}\right|$ :

$$
\left|u_{t}(x, t)\right| \leq C \frac{1}{t} \int_{\mathbf{R}^{n}} \frac{1}{|x-y|^{n-2}}\left[\left|\Delta u_{0}(y)\right|+\left|V(y) u_{0}^{p}(y)\right|\right] d y .
$$

It remains to prove that $u=u_{\infty}(x)$ is a non-trivial positive solution of the elliptic equation. But this is straightforward. Given any $\phi \in C_{0}^{\infty}\left(\mathbf{R}^{n}\right)$, we have

$$
\int_{\mathbf{R}^{n}} u(x, t) \Delta \phi(x) d x-\int_{\mathbf{R}^{n}} u_{t}(x, t) \phi(x) d x+\int_{\mathbf{R}^{n}} V(x) u^{p}(x, t) \phi(x) d x=0 .
$$

Using (4.7), (4.8), the boundedness of $u$ and the dominated convergence theorem, we obtain

$$
\int_{\mathbf{R}^{n}} u_{\infty}(x) \Delta \phi(x) d x+\int_{\mathbf{R}^{n}} V(x) u_{\infty}^{p}(x) \phi(x) d x=0 .
$$

Since, by Theorem 2.1, $u=u(x, t)$ is bounded away from zero when $u_{0} \geq \beta>0$, we know that $u_{\infty}$ is a positive solution of the elliptic equation.

Step 3. Now we assume that $V$ is an arbitary Green-tight function. We claim that there exists a sequence of smooth functions $\left\{V_{m}\right\}$ such that $V_{m} \rightarrow V$ a.e. when $m \rightarrow \infty$ and, for any domain $D \subset \mathbf{R}^{n}$,

$$
\sup _{x \in \mathbf{R}^{n}} \int_{D} \frac{\left|V_{m}(y)\right|}{|x-y|^{n-2}} d y \leq \sup _{x \in \mathbf{R}^{n}} \int_{D} \frac{|V(y)|}{|x-y|^{n-2}} d y .
$$

The proof of the claim is as follows. Let $\rho$ be the standard mollifier. Define $V_{m}(y)=\int_{|z| \leq 1} \rho(z) V\left(y-\frac{z}{m}\right) d z ;$ then we only need to check (4.9). Clearly

$$
\begin{aligned}
& \int_{D} \frac{\left|V_{m}(y)\right|}{|x-y|^{n-2}} d y \leq \int_{D} \frac{1}{|x-y|^{n-2}} \int_{|z| \leq 1} \rho(z)\left|V\left(y-\frac{z}{m}\right)\right| d z d y \\
& \quad=\int_{|z| \leq 1} \rho(z) \int_{D} \frac{\left|V\left(y-\frac{z}{m}\right)\right|}{|x-y|^{n-2}} d y d z=\int_{|z| \leq 1} \rho(z) \int_{D} \frac{|V(y)|}{\left|x-\frac{z}{m}-y\right|^{n-2}} d y d z .
\end{aligned}
$$


Hence

$$
\sup _{x \in \mathbf{R}^{n}} \int_{D} \frac{\left|V_{m}(y)\right|}{|x-y|^{n-2}} d y \leq \sup _{x \in \mathbf{R}^{n}} \int_{D} \frac{|V(y)|}{|x-y|^{n-2}} d y \int_{|z| \leq 1} \rho(z) d z .
$$

This proves the claim.

By step 2, for each $m$, there is a global positive solution $u_{m}$ of (1.1) when $V$ is replaced by the smooth function $V_{m}$. Moreover, $\lim _{t \rightarrow \infty} u_{m}(x, t)=u_{m, \infty}(x)$ pointwise. The claim about the rate of convergence of (4.7) in step 2 and (4.9) show that the convergence is uniform with respect to $m$. Theorem $\mathrm{C}$ in [Zhang2] also shows that $\left\{u_{m}\right\}$ is equicontinuous and bounded. Therefore, a subsequence, still called $\left\{u_{m}\right\}$, converges uniformly to a function $u(x, t)$ in any compact subset of $\mathbf{R}^{n} \times(0, \infty)$. Following the argument at the end of step 2, we know that $u$ is a positive solution of (1.1) and $u=u(x, t)$ converges pointwise (as $t \rightarrow \infty$ ) to a $u_{\infty}$ which is a positive solution of (1.2). q.e.d.

\section{Appendix}

The objective of the section is to prove the inequality that was used to obtain (3.2) in section 3. This inequality was first proved in [Zhang2]. Since that paper has yet to appear, we give the proof here for completeness.

Lemma 5.1. Suppose $0<a<b$. Then there exist positive constants $C_{a, b}$ and $c$ depending only on $a$ and $b$ such that

$$
\begin{aligned}
& \text { (i). } \int_{s}^{t} \int_{\mathbf{R}^{n}} G_{a}(x, t ; z, \tau)|V(z, \tau)| G_{b}(z, \tau ; y, s) d z d \tau \leq C_{a, b} N_{c, \infty}(V) G_{a}(x, t ; y, s) ; \\
& \text { (ii). } \int_{s}^{t} \int_{\mathbf{R}^{n}} G_{b}(x, t ; z, \tau)|V(z, \tau)| G_{a}(z, \tau ; y, s) d z d \tau \leq C_{a, b} N_{c, \infty}(V) G_{a}(x, t ; y, s) .
\end{aligned}
$$

Remark 5.1. The condition $a<b$ is indispensable for Lemma 5.1.

Proof of the Lemma. We will only give a proof of (i), since the proof of (ii) is similar. For simplicity we write

$$
J(x, t ; y, s)=\int_{s}^{t} \int_{\mathbf{R}^{n}} G_{a}(x, t ; z, \tau)|V(z, \tau)| G_{b}(z, \tau ; y, s) d z d \tau .
$$

Clearly we can assume that $s=0$, and hence we only need to show that

$$
J(x, t ; y, 0) \leq C N_{c, \infty}(V) G_{a}(x, t ; y, 0) .
$$

For some $\rho \in(0,1)$ to be chosen later, we have

$$
\begin{aligned}
J(x, t ; y, 0) & =\int_{0}^{\rho t} \int_{\mathbf{R}^{n}} G_{a}|V| G_{b} d z d \tau+\int_{\rho t}^{t} \int_{\mathbf{R}^{n}} \ldots d z d \tau \\
& \equiv J_{1}+J_{2} .
\end{aligned}
$$

We will estimate $J_{1}$ first. To this end let us recall the inequality

$$
\frac{|x-z|^{2}}{t-\tau}+\frac{|z-y|^{2}}{\tau-s} \geq \frac{|x-y|^{2}}{t-s}, \quad 0<s<\tau<t .
$$


By (5.3) we know that

$$
\begin{aligned}
J_{1} & =\int_{0}^{\rho t} \int_{\mathbf{R}^{n}} \frac{\exp \left(-a \frac{|x-z|^{2}}{t-\tau}\right)}{(t-\tau)^{n / 2}}|V(z, \tau)| \frac{\exp \left(-b \frac{|z-y|^{2}}{\tau}\right)}{(\tau)^{n / 2}} d z d \tau \\
& =\int_{0}^{\rho t} \int_{\mathbf{R}^{n}} \frac{\exp \left(-a\left[\frac{|x-z|^{2}}{t-\tau}+\frac{|z-y|^{2}}{\tau}\right]\right)}{(t-\tau)^{n / 2}}|V(z, \tau)| \frac{\exp \left(-(b-a) \frac{|z-y|^{2}}{\tau}\right)}{(\tau)^{n / 2}} d z d \tau .
\end{aligned}
$$

By (5.4),

$$
\exp \left(-a\left[\frac{|x-z|^{2}}{t-\tau}+\frac{|z-y|^{2}}{\tau}\right]\right) \leq \exp \left(-a \frac{|x-y|^{2}}{t}\right) .
$$

Moreover, $t-\tau \geq(1-\rho) t$ for $\tau \in(0, \rho t)$. Therefore (5.5) implies

$$
J_{1} \leq \frac{\exp \left(-a \frac{|x-y|^{2}}{t}\right)}{((1-\rho) t)^{n / 2}} \int_{0}^{\rho t} \int_{\mathbf{R}^{n}}|V(z, \tau)| \frac{\exp \left(-(b-a) \frac{|z-y|^{2}}{\tau}\right)}{(\tau)^{n / 2}} d z d \tau,
$$

which means that

$$
J_{1} \leq(1-\rho)^{-n / 2} N_{b-a, \infty}(V) G_{a}(x, t ; y, 0) .
$$

Next we estimate $J_{2}$. From (3.3) we have

$$
\begin{aligned}
J_{2} & =\int_{\rho t}^{t} \int_{\mathbf{R}^{n}} \frac{\exp \left(-a \frac{|x-z|^{2}}{t-\tau}\right)}{(t-\tau)^{n / 2}}|V(z, \tau)| \frac{\exp \left(-b \frac{|z-y|^{2}}{\tau}\right)}{(\tau)^{n / 2}} d z d \tau \\
& =\int_{\rho t}^{t} \int_{|z-y| \geq|x-y|(a / b)^{1 / 2}} \ldots d z d \tau+\int_{\rho t}^{t} \int_{|z-y| \leq|x-y|(a / b)^{1 / 2}} \ldots d z d \tau \\
& \equiv J_{21}+J_{22} .
\end{aligned}
$$

When $|z-y| \geq|x-y|(a / b)^{1 / 2}$ and $\tau \in(\rho t, t)$, then

$$
\frac{\exp \left(-b \frac{|z-y|^{2}}{\tau}\right)}{(\tau)^{n / 2}} \leq \frac{\exp \left(-a \frac{|x-y|^{2}}{t}\right)}{(\rho t)^{n / 2}}
$$

Therefore

$$
J_{21} \leq \frac{\exp \left(-a \frac{|x-y|^{2}}{t}\right)}{(\rho t)^{n / 2}} \int_{\rho t}^{t} \int_{|z-y| \geq|x-y|(a / b)^{1 / 2}} \frac{\exp \left(-a \frac{|x-z|^{2}}{t-\tau}\right)}{(t-\tau)^{n / 2}}|V(z, \tau)| d z d \tau,
$$

which gives

$$
J_{21} \leq(\rho)^{-n / 2} N_{a, \infty}(V) G_{a}(x, t ; y, 0) .
$$

Finally we will estimate $J_{22}$. From (3.7), we have

$$
J_{22} \leq(\rho t)^{-n / 2} \int_{\rho t}^{t} \int_{|z-y| \leq|x-y|(a / b)^{1 / 2}} \frac{\exp \left(-a \frac{|x-z|^{2}}{t-\tau}\right)}{(t-\tau)^{n / 2}}|V(z, \tau)| d z d \tau .
$$

If $|z-y| \leq|x-y|(a / b)^{1 / 2}$, then

$$
|x-z| \geq|x-y|-|z-y| \geq|x-y|\left(1-(a / b)^{1 / 2}\right) .
$$


Hence

$$
\begin{aligned}
\exp \left(-a \frac{|x-z|^{2}}{t-\tau}\right) & =\exp \left(-a \frac{|x-z|^{2}}{2(t-\tau)}\right) \exp \left(-a \frac{|x-z|^{2}}{2(t-\tau)}\right) \\
& \leq \exp \left(-a \frac{|x-z|^{2}}{2(t-\tau)}\right) \exp \left(-a \frac{|x-y|^{2}}{2(t-\tau)}\left(1-(a / b)^{1 / 2}\right)^{2}\right) \\
& \leq \exp \left(-a \frac{|x-z|^{2}}{2(t-\tau)}\right) \exp \left(-a \frac{|x-y|^{2}}{2(1-\rho) t}\left(1-(a / b)^{1 / 2}\right)^{2}\right)
\end{aligned}
$$

Here we have used the fact that $0<t-\tau \leq(1-\rho) t$. Now taking $\rho$ so that

$$
\frac{\left(1-(a / b)^{1 / 2}\right)^{2}}{2(1-\rho)}=1
$$

we obtain,

$$
\exp \left(-a \frac{|x-z|^{2}}{t-\tau}\right) \leq \exp \left(-a \frac{|x-z|^{2}}{2(t-\tau)}\right) \exp \left(-a \frac{|x-y|^{2}}{t}\right)
$$

Substituting (5.11) in (5.9), we have

$$
J_{22} \leq \frac{\exp \left(-a \frac{|x-y|^{2}}{t}\right)}{(\rho t)^{n / 2}} \int_{\rho t}^{t} \int_{|z-y| \leq|x-y|(a / b)^{1 / 2}} \frac{\exp \left(-a \frac{|x-z|^{2}}{2(t-\tau)}\right)}{(t-\tau)^{n / 2}}|V(z, \tau)| d z d \tau
$$

which yields

$$
J_{22} \leq(\rho)^{-n / 2} N_{a / 2, \infty}(V) G_{a}(x, t ; y, 0) .
$$

Combining (5.8) and (5.12), we have

$$
J_{2} \leq 2(\rho)^{-n / 2} N_{a / 2, \infty}(V) G_{a}(x, t ; y, 0) .
$$

Inequalities (5.6) and (5.13) imply (5.2) with $c=\min \{b-a, a / 2\}$ and the lemma. q.e.d.

\section{REFERENCES}

[A] D.G. Aronson, Non-negative solutions of linear parabolic equations, Annali della Scuola Norm. Sup. Pisa 22 (1968), 607-694. MR 55:8553

[AS] M. Aizenman and B. Simon, Brownian motion and Harnack's inequality for Schrödinger operators, Comm. Pure Appl. Math 35 (1982), 209-271. MR 84a:35062

[CFG] F. Chiarenza, E. Fabes and N. Garofalo, Harnack's inequality for Schrödinger operators and the continuity of solutions, Proc. Amer. Math. Soc. 98 (1986), 415-425. MR 88a:35037

[F] H. Fujita, On the blowing up of solutions of the Cauchy problem for $u_{t}=\Delta u+u^{1+\alpha}$, J. Fac. Sci. Univ. Tokyo, Sect I 13 (1966), 109-124. MR 35:5761

[KN] C. Kenig and W. M. Ni, An exterior Dirichlet problem with applications to some equations arising in geometry, Amer. J. Math. 106 (1984), 689-702. MR 85j:35072

[L] F. H. Lin, On the elliptic equation $D_{i}\left[a_{i j}(x) D_{j} U\right]-k(x) U^{p}=0$, Proc, AMS 95 (1985), 219-266. MR 86k:35041

[N] W. M. Ni, On the elliptic equation $\Delta v+K(x) u^{(n+2) /(n-2)}=0$, its generalizations and applications in geometry, Indiana Univ. Math. J. 31 (1982), 493-529. MR 84e:35049

[Zhang1] Qi Zhang, On a parabolic equation with a singular lower order term, Transactions of AMS 348 (1996), 2811-2844. MR 96k:35073

[Zhang2] Qi Zhang, Global existence and local continuity of solutions for semilinear parabolic equations, Comm. PDE, to appear 1997. 
[Zhang3] Qi Zhang, Linear parabolic equations with singular lower order coefficients, PhD Thesis, Purdue U. 1996.

[Zhao1] Z. Zhao, On the existence of positive solutions of nonlinear elliptic equations- A probabilistic potential theory approach, Duke Math J. 69 (1993), 247-258. MR 94c:35090

[Zhao2] Z. Zhao, Subcriticality, positivity, and guageability of the Schrödinger operator, Bull. AMS 23 (1990), 513-517. MR 91h:35104

Department of Mathematics, University of Missouri, Columbia, Missouri 65211

E-mail address: sz@math.missouri.edu

E-mail address: zzhao@math.missouri.edu 